\title{
Erratum to: Severe somatoform and dysautonomic syndromes after HPV vaccination: case series and review of literature
}

\author{
Beniamino Palmieri ${ }^{1}$ - Dimitri Poddighe ${ }^{2}$. \\ Maria Vadalà $^{\mathbf{1}} \cdot$ Carmen Laurino $^{1}$. \\ Carla Carnovale $^{3} \cdot$ Emilio Clementi $^{3}$
}

Published online: 20 September 2016

(C) Springer Science+Business Media New York 2016

\section{Erratum to: Immunol Res \\ DOI 10.1007/s12026-016-8820-z}

The original version of this article unfortunately contained a mistake. It has been corrected with this erratum. And the original article was also corrected.

1. The co-corresponding author of the article is Dr. Dimitri Poddighe (dimimedpv@yahoo.it).

2. Prof. Beniamino Palmieri and Dr. Dimitri Poddighe contributed equally to the drafting and writing of this study.

3. The correct version of Tables 3 and 5 are given below.
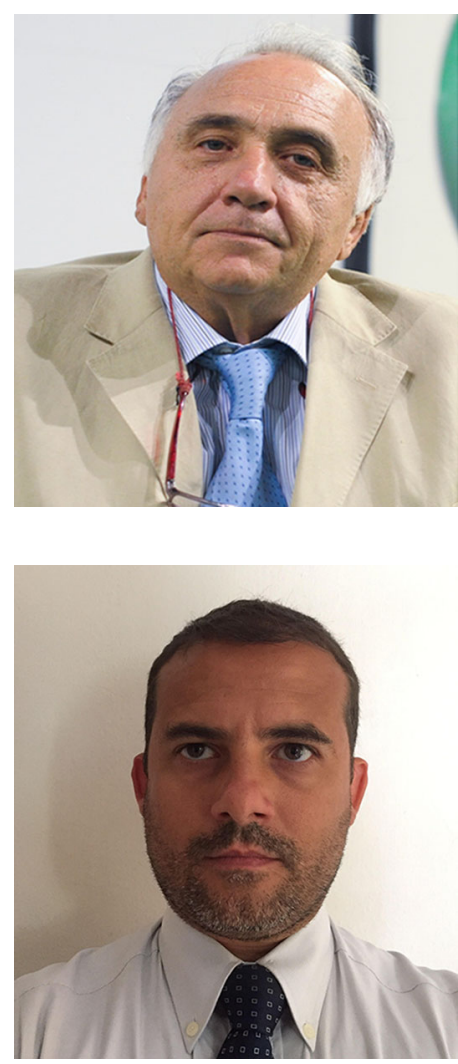

Beniamino Palmieri Dimitri Poddighe

Prof. Beniamino Palmieri and Dr. Dimitri Poddighe contributed equally to the drafting and writing this study.

The online version of the original article can be found under doi:10. 1007/s12026-016-8820-z.

Beniamino Palmieri

palmieri@unimore.it

$\triangle$ Dimitri Poddighe

dimimedpv@yahoo.it

1 Department of General Surgery and Surgical Specialties, University of Modena and Reggio Emilia Medical School, Surgical Clinic, Modena, Italy

2 Department of Paediatrics, ASST Melegnano e Martesana, Vizzolo Predabissi, MI, Italy

3 Unit of Clinical Pharmacology, Department of Biomedical and Clinical Sciences, National Research Council-Institute of Neuroscience, University Hospital L. Sacco, University of Milano, Milan, Italy 
Table 3 Description of ADRs related to HPV immunization

After first dose

After second dose

After third dose

Symptoms (within 5 days post-vaccination)

Injection site reactions

Pain

Uncontrollable and involuntary movement of the limb

Swelling

Systemic reactions

Low-grade fever

Headache

Recurrent syncope

Persistent convulsive hunger

Irritability

Epileptic seizures

Temporary speech loss

Lower limbs paraesthesia and paresis

Hot flushes

Severe stomach pain

Insomnia

Hypersensitivity reactions

Leg muscle pain (myalgia)

Gait and orthostatic posture impairment

Excessive sweating

Vomiting

Symptoms (5-15 days post-vaccination)

Systemic reactions

Asthenia

Persistent thirst

Severe hands and feet itching

Optic neuritis

\section{Symptoms (15-20 days post-vaccination)}

Systemic reactions

Amenorrhoea

Skin rashes

Tachycardia

Difficult breathing

Weight loss
2

1

2

1

1

1

1

1

2

1

3

1

1

1

5

3

1

1

1

1

1

1
1 
Table 5 Proposed diagnostic criteria of autoimmune/inflammatory syndrome induced by adjuvants (ASIA)

\section{Major criteria}

Previous exposure to an external stimulus (i.e. vaccine, adjuvant, silicone, nucleic acids, fragments of bacterial cell walls)

One of the following "typical" manifestations:

Myalgia/myositis, muscle weakness

Arthralgia and/or arthritis

Chronic fatigue, non-refreshing sleep or sleep disturbances

Neurological manifestations (especially if associated with demyelination)

Memory loss and cognitive impairment

Fever

Dry mouth

Improvement of symptoms after the removal of the triggering agent

Typical biopsy of the involved organs

\section{Minor criteria}

Appearance of autoantibodies or autoantibodies directed against the suspected adjuvant

Other clinical manifestations (i.e. functional somatic syndromes)

Association with specific HLA haplotypes (i.e. HLA-DRB1, HLA-DQB1)

Development of autoimmune diseases

\section{Diagnostic requirements:}

Two major criteria

One major criteria + two minor criteria 